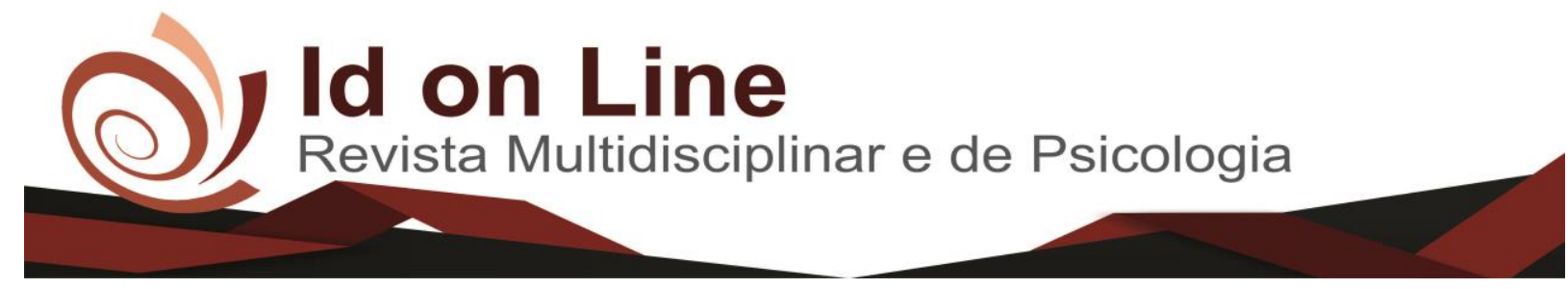

Artigo

\title{
Capacidade funcional de idosos em instituição de longa permanência
}

\author{
Camila Santos Silva ${ }^{1}$,Lucas Silveira Sampaio²; Talita Santos Oliveira Sampaio ${ }^{3}$
}

\begin{abstract}
Resumo: A capacidade funcional diz respeito a possibilidade de realização das Atividades de Vida Diária, e este é o mais significativo indicador de que o idoso permanece funcional em sua rotina. Nesse sentido, o presente trabalho tem por objetivo avaliar a capacidade funcional de idosos institucionalizados em uma única instituição de longa permanência para idosos no município de Itambé. A pesquisa possui caráter descritivo-exploratório e é de natureza quantitativa possuindo delineamento transversal. Foi utilizado um questionário para identificação dos aspectos sociodemográficos dos idosos e a Escala de Katz para capacidade funcional. Os dados foram tabulados através do software Excel 2013 e foram analisados no The Statistical Package for Social Sciences para Windows. Por meio do estudo, identificou-se que a média de idade da população foi de 75,2 anos, $70 \%$ eram do sexo feminino, $80 \%$ eram analfabetos, $70 \%$ dos pesquisados se referiram solteiros e $100 \%$ possuíam filhos. Em relação aos aspectos da institucionalização, $50 \%$ dos idosos foram encaminhados para a instituição através do Centro de Referência Especializado de Assistência Social e 50\% dos residentes habitavam há mais de cinco anos naquele local. Em relação à capacidade funcional, observou-se que $60 \%$ dos idosos institucionalizados eram dependentes. Diante dos resultados, sugere-se que a institucionalização pode trazer aspectos negativos na saúde do idoso sendo necessário ações que visem a prevenção da alteração da capacidade funcional.
\end{abstract}

Palavras-chave: Saúde do Idoso. Instituição de Longa Permanência para Idosos. Assistência a idosos.

\section{Functional capacity of the elderly in a long-term institution}

\begin{abstract}
Functional capacity refers to the possibility of performing daily life activities, and this is the most significant indicator that the elderly remain functional in their routine. In this sense, this study aims to evaluate the functional capacity of institutionalized elderly in a single long-term institution for the elderly in the city of Itambé. The research has a descriptive-exploratory character and is quantitative in nature having cross-sectional design. A questionnaire was used to identify the sociodemographic aspects of the elderly and the Katz Scale for functional capacity. The data were tabulated using Excel 2013 software and analyzed in The Statistical Package for Social Sciences for Windows. Through the study, identified that the mean age of the population was 75.2 years, $70 \%$ were female, $80 \%$ were illiterate, $70 \%$ of those surveyed reported being single, and $100 \%$ had children. Regarding institutionalization aspects, $50 \%$ of the elderly were referred to institution through the Center of Reference in Specialized Social Assistance and $50 \%$ of the residents lived in that place for more than five years. Regarding functional capacity, it was observed that $60 \%$ of the institutionalized elderly were dependent. In view of the results, it is suggested that the institutionalization can bring negative aspects in the health of the elderly, being necessary actions that aim at the prevention of the alteration of functional capacity.
\end{abstract}

Keywords: Elderly Health. Long-Staying Institution for the Elderly. Elderly Assistance.

\footnotetext{
1Discente do curso de Fisioterapia na Faculdade Independente do Nordeste (FAINOR). Contato: milla-ssantos@ hotmail.com.

${ }^{2}$ Docente do curso de Fisioterapia na Faculdade Independente do Nordeste (FAINOR). Mestre em ciências da saúde pela Universidade Estadual do Sudoeste da Bahia (UESB). Contato: lucassilveira@ fainor.com.br

${ }^{3}$ Docente do curso de Fisioterapia na Faculdade Independente do Nordeste (FAINOR). Mestre em ciências da saúde pela Universidade Estadual do Sudoeste da Bahia. Contato: talitafisio@gmail.com
} 


\section{Introdução}

Segundo dados da Secretaria de Direitos Humanos apoiados em estatísticas demográficas da Organização das Nações Unidas (ONU), aproximadamente 10\% das pessoas do mundo tem mais de 60 anos. Projeções recentes apontam que até o ano de 2050, a população mundial de idosos superará a de crianças menores de 15 anos. No Brasil, segundo o último censo demográfico do Instituto Brasileiro de Geografia e Estatística - IBGE, a população idosa totaliza, atualmente, 23,5 milhões de pessoas (BRASIL, 2012).

Entende-se por idoso, aquele indivíduo que tenha idade superior ou igual a 60 (sessenta) anos e que, de modo natural, esteja enfrentando o processo de envelhecimento, que diz respeito às mudanças biológicas, psicológicas e sociais (BRASIL, 2003; SANTOS, 2010). O conceito de envelhecimento está relacionado com a dinamicidade de um processo contínuo no qual há mudanças funcionais, bioquímicas, morfológicas e psicológicas, que vão determinar a perda gradativa de adaptar-se ao ambiente, estando o indivíduo mais vulnerável a doenças crônicas degenerativas e ao óbito de forma precoce (FREITAS et al., 2006).

As doenças crônicas degenerativas, tais como diabetes mellitus, (Acidente Vascular Encefálico) e doenças cardíacas, juntamente com todas as alterações fisiológicas esperadas para o indivíduo idoso, como diminuição da capacidade do sistema cardiovascular, diminuição da função pulmonar, envelhecimento musculoesquelético (perda de massa muscular, da viscosidade dos fluidos e elasticidade dos tendões), comprometido do Sistema Nervoso Central (SNC), além do envelhecimento psicológico e social, podem afetar a capacidade do idoso em realizar as suas atividades cotidianas, ou seja a sua capacidade funcional (BARBOSA et al., 2014; FECHINE; TROMPIERI, 2012)

A capacidade funcional diz respeito a capacidade de realização das Atividades de Vida Diária (AVD) e é o mais significativo indicador de que o idoso permanece funcional no cotidiano. O envelhecimento vai gerar, naturalmente, alterações nas AVD e são vários os fatores que levam a essa diminuição da funcionalidade como, por exemplo, aspectos sociodemográficos e socioambientais, bem como também, a institucionalização (LOPES; SANTOS, 2015).

A institucionalização do idoso é uma realidade cada vez mais presente no Brasil. Dentre as principais causas, encontra-se o aumento da participação das mulheres no mercado de trabalho o que a impossibilita, muitas vezes, de os familiares dedicarem tempo ao cuidado de 
seus idosos. A permanência nessas instituições pode gerar impactos negativos na condição de saúde, qualidade de vida e funcionalidade do idoso. Vale ressaltar que a institucionalização está quase sempre atrelada ao abandono, aos que perderam suas famílias ou a vulnerabilidade social. Portanto, a vivência ali pode influenciar no desempenho das atividades de vida diária (AVD) (BASSLER et al., 2016; CAMARANO; KANSO, 2010).

Segundo Santiago e Mattos (2014) a prevalência de alteração da capacidade funcional do idoso institucionalizado é maior, quando comparada com estudos de outras populações, visto que fatores associados a institucionalização, idade elevada, comorbidades, entre outros, agravam a funcionalidade. Corroborando Gonçalves (2010) já havia percebido esse fenômeno em estudo realizado em três regiões do país, concluindo que a institucionalização e a consequente dependência contribuem para a prevalência de alteração da capacidade funcional do idoso.

Diante deste contexto, torna-se importante a realização deste estudo em detrimento do aumento do número de idosos nas sociedades e dos impactos negativos que a perda da capacidade funcional pode gerar na qualidade de vida dos idosos institucionalizados. Destacase que o estudo busca contribuir no conhecimento maior dos pesquisadores, profissionais de saúde e sociedade em geral sobre a funcionalidade dos idosos institucionalizados, visando medidas preventivas contra a incapacidade dessa população.

Sendo assim, este estudo tem por objetivo avaliar a capacidade funcional de idosos institucionalizados.

\section{Métodos}

Trata-se de um estudo descritivo-exploratório, com delineamento transversal e abordagem quantitativa. A pesquisa de natureza quantitativa diz respeito aos elementos passiveis de serem quantificados, além disso centra-se na objetividade. Para Rodrigues e Limena (2006) a pesquisa quantitativa é compreendida como aquela que quantifica os dados a fim de analisá-los estando voltado para análise e a interpretação dos resultados.

O estudo foi realizado em uma instituição de longa permanência para idosos da cidade de Itambé-BA. Este munícipio está localizado no sudoeste da Bahia. A população da pesquisa 
foi constituída por todos os idosos, de ambos os sexos, residentes na instituição de longa permanência, totalizando 10 idosos.

Os dados foram coletados por meio de um questionário sociodemográfico adaptado, o qual apurou informações dos prontuários dos idosos como sexo, idade, estado civil, escolaridade, entre outras informações. Já para a avaliação da capacidade funcional foi aplicada a escala de Katz, objetivando mensurar os níveis individuais de cada sujeito em independentes ou não, do seu próprio cuidado, contendo 6 itens como: banhar-se, vestir-se, ir ao banheiro, transferência, continência e alimentar-se (KATZ; AKPOM, 1976).

Nesta avaliação foi identificado o índice de dependência e independência sem ajuda: AIndependente para comer, banhar-se, ser continente, mobilizar-se, usar o sanitário e vestir-se; B - Independente para realizar todas estas funções exceto uma; C- independência pra realizar todas as funções, exceto banhar-se e outra função mais; D- Independente para realizar todas as funções, exceto para banhar-se, vestir-se e outra função mais; E - Independente para realizar todas as funções, exceto banhar-se, vestir-se, usar o sanitário; F - Independente para realizar todas as funções, exceto banhar-se, vestir-se, usar o sanitário, mobilizar-se e outra função mais, $\mathrm{G}$ - dependente para realizar as seis funções. Sendo classificada do seguinte modo: 6 pontos representa independente; 4 - dependência moderada; 2 ou menos - muito dependente. No presente estudo foi classificado como independente aqueles com valor $\geq 6$ pontos e como dependentes aqueles que possuíam valor $\leq 4$ pontos (SMANIOTO et al., 2011).

Os dados foram tabulados no programa Excel for Windows, versão 2013. Para a análise descritiva das características da população foi calculado a frequência absoluta. Os dados foram analisados no The StatisticalPackage for Social Sciences para Windows (SPSS 22.0, 2013, SPSS, Inc, Chicago, IL).

Essa pesquisa respeita às normas éticas exigidas pela Resolução n 466/2012 (Conselho Nacional de Saúde), que incluem a aplicação do Termo de Consentimento Livre e Esclarecido - TCLE assinado por cada participante (APÊNDICE A). O protocolo foi submetido ao Comitê de Ética em Pesquisa com Seres Humanos da Faculdade Independente do Nordeste (CEPFAINOR) e aprovado com o parecer de número 2261873. 


\section{Resultados}

A partir da análise dos dados, identificou-se que a idade média dos participantes do estudo foi de 75,2 $\pm 10,29$ anos, sendo que $70 \%$ eram do sexo feminino $(n=7)$ e $80 \%(n=8)$ dos idosos eram analfabetos. Em relação ao estado civil, 70\% dos indivíduos referiram ser solteiros $(\mathrm{n}=7)$. Destaca-se ainda que $100 \%(\mathrm{n}=6)$ possuíam filhos vivos.

Tabela 1. Características sociodemográficas dos participantes da pesquisa. Itambé-Ba, 2017.

\begin{tabular}{lccc}
\hline Variáveis & \% resposta & $\mathrm{N}$ & $\%$ \\
\hline Sexo & 100 & & \\
$\quad$ Masculino & & 3 & 30 \\
$\quad$ Feminino & 100 & 7 & 70 \\
Escolaridade & & 2 & 20 \\
$\quad$ Alfabetizado & & 8 & 80 \\
$\quad$ Analfabeto & 100 & & 70 \\
Estado Civil Atual & & 7 & - \\
$\quad$ Solteiro & & - & 20 \\
$\quad$ Casado & & 2 & 10 \\
$\quad$ Viúvo & & 1 & 100 \\
$\quad$ Separado & 60 & & - \\
Filhos & & 6 & \\
Sim & & - & \\
Não & & & \\
\hline
\end{tabular}

Fonte: Dados da pesquisa

Em relação aos aspectos da institucionalização, 50\% dos idosos foram encaminhados para a institucionalização através do Centro de Referência Especializado de Assistência Social (CREAS) e $30 \%$ foram conduzidos a este processo por algum familiar. Em relação ao tempo em que estavam institucionalizados, $50 \%$ já possuíam mais de cinco anos no local, sendo uma média de institucionalização de 5,4 $\pm 3,47$ anos. 
Tabela 2. Descrição dos aspectos relacionados ao processo de institucionalização. ItambéBa, 2017.

\begin{tabular}{lccc}
\hline Variáveis & \% resposta & N & \% \\
\hline Encaminhamento & 100 & & \\
Familiar & & 3 & 30 \\
Cuidador & & 1 & 10 \\
Decisão própria & & 1 & 10 \\
Assistência Social/CREAS & 5 & 50 \\
Tempo de Institucionalização & 100 & & \\
$\quad<5$ anos & & 5 & 50 \\
$>5$ anos & & 5 & 50 \\
\hline
\end{tabular}

Fonte: Dados da pesquisa.

Em relação a capacidade funcional dos idosos institucionalizados, observou-se que $60 \%$ eram dependentes para a realização das atividades da vida diária (AVD) e 20\% eram independentes.

Tabela 3. Análise descritiva da variável qualitativa Capacidade Funcional. Itambé-Ba, 2017.

\begin{tabular}{lccc}
\hline Variáveis & \% resposta & $\mathrm{N}$ & $\%$ \\
\hline Capacidade Funcional & 100 & & \\
Independente & & 4 & 40 \\
Dependente & & 6 & 60 \\
\hline
\end{tabular}

Fonte: Dados da pesquisa.

\section{Discussão}

A partir da identificação dos dados sociodemográficos foi possível verificar que houve uma predominância do sexo feminino entre os idosos institucionalizados, totalizando $70 \%$ da amostra. De acordo com Araújo, Neto e Bós (2016), o processo de institucionalização é mais presente entre as idosas em decorrência da viuvez e do receio apresentado pelas mulheres em não preocupar os filhos. Outros estudos analisam que a maior prevalência de idosos em instituições de longa permanência ser do sexo feminino, se deve a feminilização da população idosa em consequência do maior número de mulheres se comparado com o número de homens e também pelo das mulheres serem menos expostas a fatores de risco como o tabagismo e o álcool (FLEIG; OLIVEIRA, 2017; REIS et al., 2013). 
De acordo com Pinheiro et al. (2016), alguns fatores como ser solteiro, não possuir filhos e possuir grau de escolaridade baixo ou nulo podem favorecer o processo de institucionalização o que corrobora com o presente estudo. Entretanto, os resultados deste estudo apontam que $60 \%$ dos idosos possuíam filhos. Isso se torna um indicador da falta de preparo familiar na atenção à saúde e aos cuidados dos idosos que, mesmo com filhos, foram institucionalizados.

Com relação aos processos de institucionalização, o encaminhamento no presente estudo foi realizado em sua maioria pelo CREAS (Centro de Referência Especializado de Assistência Social), sendo $50 \%$ dos idosos encaminhados por essa unidade pública. O CREAS oferta serviço especializado a famílias e indivíduos em situação de ameaça ou violação dos direitos (PEREIRA, et al., 2011). Cabe ressaltar que 30\% dos idosos foram encaminhados para a instituição de longa permanência por um familiar. Um estudo realizado por Cardoso (2016) com 43 idosos pontua que o rompimento dos vínculos é o principal motivo de inserção do idoso no contexto institucional. Lima et al. (2013) relata que as famílias se sentem impossibilitadas de atender idosos diante de suas limitações e o cuidado se torna ainda mais difícil quando algumas patologias estão presentes, sendo assim, a institucionalização é o meio de proporcionar melhores condições de cuidado.

De acordo com o tempo de institucionalização, $50 \%$ dos idosos foram institucionalizados há mais de 5 anos. De acordo com Oliveira e Mattos (2010) em seu estudo com 154 idosos 84,4\% dos mesmos se encontravam há menos de 4 anos na instituição. Nobre (2016) também evidenciou em seu estudo realizado com 44 idosos, que 77,27\% destes, apresentavam tempo de institucionalização inferior a 4 anos. Segundo Borges e Mende (2016) o tempo de permanência dos idosos nas instituições é proporcional à capacidade funcional dessa população. Ou seja, quanto maior o tempo de institucionalização, maior será o declínio da capacidade funcional desses idosos.

No que diz respeito à avaliação da capacidade funcional dos idosos entrevistados, constatou-se que $60 \%$ dos idosos apresentava dependência para realizar atividades de vida diária. Estudo realizado por Carlos, Gazzola e Gomes (2016) evidenciaram que a maior parte da amostra (83,3\%), composta por 24 idosos institucionalizados, também apresentaram declínio da capacidade funcional. Chaves et al (2017) em seu estudo com 20 idosos constatou que $65 \%$ dos idosos apresentavam dependência moderada corroborando com o presente estudo, já Costa et al (2017) comparou idosos institucionalizados e não institucionalizados e demonstrou que 56,8\% dos idosos que residiam na ILP se encontravam com alguma dependência, com resultados que 
corroboram com o presente estudo. No entanto, Antonelli et al. (2017) realizaram um estudo avaliando a capacidade funcional de 15 idosos em uma instituição de longa permanência e evidenciaram que a maior parte da população estudada $(57,3 \%)$ apresentou boa capacidade para realizar as atividades básicas de vida diária.

Esses resultados podem ser atribuídos à transição para o ambiente de institucionalização, uma vez que este envolve aspectos psicológicos como o estresse emocional, a separação da família e o abandono, contribuindo para o aumento da dependência dos idosos.

Reis, Marinho e Lima (2014) afirmam em seu estudo que a capacidade funcional dos idosos sofre declínio fisiológico em decorrência dos comprometimentos referentes ao processo de envelhecimento, que associado ao processo de institucionalização aumenta a dependência física do idoso.

Silva et al (2015) em seu estudo afirmam que a institucionalização, a depressão e a capacidade funcional estão diretamente ligados, pois a institucionalização leva o idoso a um senso de isolamento social e consequentemente a depressão e por fim a perda da capacidade de realizar atividade de vida diária.

Segundo Guimarães e Martins (2016) as varáveis quanto a gênero feminino, grau de escolaridade baixo estão diretamente ligados ao processo de institucionalização e consequentemente quanto maior o tempo que o idoso permanecer na instituição maior será seu comprometimento funcional o que explica o resultado do presente estudo no qual a maioria dos idosos eram do sexo feminino, com baixo grau de escolaridade e tinham mais de 5 anos de institucionalização.

\section{Considerações Finais}

Diante dos resultados apresentados, é possível concluir que a institucionalização pode gerar aspectos negativos na saúde dos idosos e mostram que a maioria dos idosos apresentam dependência para realizar atividades básicas de vida diária. Dessa forma, torna-se importante a realização de novos estudos com populações maiores afim de ampliar o conhecimento acerca do tema, tanto por sua recorrência, quanto por sua importância. Além disso, salienta-se a necessidade de planejamentos de estratégias voltadas para manutenção da capacidade funcional do idoso e orientações para familiares em relação aos cuidados prestados aos idosos, a fim de prevenir a institucionalização. 


\section{Referências}

ANTONELLI, G. et al. Avaliação da capacidade funcional de idosos em uma instituição de longa permanência de Guarapuava - Paraná. Revista do Departamento de Educação Física e Saúde e do Mestrado em Promoção da Saúde da Universidade de Santa Cruz do Sul / Unisc. Ano 18 - Volume 18 - Número 3 - Julho/Setembro 2017

ARAÚJO, A. M.; NETO, T. B. S. de.; BÓS, A. J. G. Diferenças no perfil de pessoas idosas institucionalizadas, em lista de espera e que não desejam institucionalização. Rev. Bras. Geriatr. Gerontol. Rio de Janeiro, 2016; 19(1):105-118

BRASIL. Dados sobre o envelhecimento no Brasil. Disponível em: $<$ http://www.sdh.gov.br/assuntos/pessoa-idosa/dados estatisticos/Dadossobreoenvelhecimento noBrasil.pdf>. Acesso em 12.03.2017.

BASSLER, Thais Carolina et al. O Perfil Nutricional de Idosos Institucionalizados num Município do Interior do Mato Grosso Do Sul. Acta de Ciências e Saúde, v. 1, n. 2, p. 1-14, 2016.

BARBOSA, Bruno Rossi et al. Avaliação da capacidade funcional dos idosos e fatores associados à incapacidade. Revista Ciência \& Saúde Coletiva, v. 19, n. 8, 2014.

BORGES, A. P. M.; MENDES, G. C. Avaliação cognitiva e de equilíbrio em idosos institucionalizados após intervenção de xbox terapia. Cad. da Esc. de Saúde, Curitiba, V.1 N.13: 93-104

CARLOS, A. G.; GAZZOLA, J. M.; GOMES, A. C. de. Funcionalidade de Idosos Institucionalizados: a Influência do Estado Nutricional. Rev. Equilíbrio Corporal Saúde 2016;8(1):17-22, 2016

CAMARGOS, Mirela Castro Santos. Instituições de longa permanência para idosos: um estudo sobre a necessidade de vagas. Revista Brasileira de Estudos de População, v. 31, n. 1, p. 211-217, 2014.

COSTA, F. N. Comparação do Estado Nutricional, Qualidade de vida e Capacidade Funcional entre Idosos Institucionalizados e não Institucionalizados. Universidade estadual paulista Júlio de mesquita filho faculdade de medicina, 2017.

CHAVES, R.N.et al. Perda Cognitiva e Dependência Funcional em Idosos Longevos Residentes em Instituições de Longa Permanência. Revista cogitaire enfermagem, v.22, n.1, 2017.

FECHINE, Basílio Rommel Almeida; TROMPIERI, Nicolino. O Processo de Envelhecimento: as Principais Alterações que acontecem com o idoso com o passar dos anos. InterSciencePlace, v. 1, n. 20, 2015.

FLEIG, T. C. M.; OLIVEIRA, M. R. Avaliação da funcionalidade de idosos institucionalizados: relação entre a MIF e a ICF. Revista do Departamento de Educação Física e Saúde e do Mestrado em Promoção da Saúde da Universidade de Santa Cruz do Sul / Unisc. Ano 18 - Volume 18 - Número 3 - Julho/setembro 2017

FREITAS, Elizabete Viana de et al. Tratado de geriatria e gerontologia. Rio de Janeiro: Guanabara Koogan, 2006.

GONCALVES, Lúcia Hisako Takase et al. O idoso institucionalizado: avaliação da capacidade funcional e aptidão física. Cad. Saúde Pública. vol.26, n.9, pp.1738-1746, 2010. 
IBGE - Instituto Brasileiro de Geografia e Estatística. Síntese de indicadores sociais: uma análise das condições de vida da população brasileira. Disponível em: <http://biblioteca.ibge.gov.br/visualizacao/livros/liv98965.pdf>. Acesso em mar. de 2017

KATZ, S.; AKPOM C.A. A measure of primary sociobiological functions. Int J Health Serv, v.6, n.3, p. 493-508. 1976.

LIMA, C.L.J et.al. Perfil sociodemografico e clinico de idosos institucionalizados. Revista de enfermagem UFPE online. Recife outubro (2013)

LOPES, Geovanna Lemos; SANTOS, Maria Izabel Penha de Oliveira. Funcionalidade de idosos cadastrados em uma unidade da Estratégia Saúde da Família segundo categorias da Classificação Internacional de Funcionalidade. Rev. bras. geriatr. gerontol, v. 18, n. 1, p. 71-83, 2015.

OLIVEIRA, P.H.; MATTOS, I.E., Prevalencia e Fatores Associados a Incapacidade Funcional em Idosos Institucionalizados no Municipio de Cuiaba, Estado De Mato Grosso, 2009/2010.

ONU - Organização das Nações Unidas. A ONU e as pessoas idosas. Disponível em: $<$ https://nacoesunidas.org/acao/pessoas-idosas/>. Acesso em maio de 2017.

PINHEIRO, M. C. G., et al. Desigualdade no perfil dos idosos institucionalizados na cidade de Natal, Brasil. Ciênc. saúde coletiva vol.21 no.11 Rio de Janeiro Nov. 2016

REIS, L.A.dos. et al. Perfil Sociodemográfico e de Saúde do idoso em instituição de longa permanência para idosos em Vitória da Conquista Bahia. Revista InterScientia, Joao Pessoa, v.1, n. 3, p.50-59, set/dez.2013

REIS, L. A. dos.; MARINO, M. S. dos.; LIMA, P. V. comprometimento da capacidade funcional: Significados para o idoso e sua família. Revista InterScientia, João Pessoa, v.2, n.1, p. 108-121, jan./abr. 2014

Silva, J.K.S., et al. (2015). Sintomas Depressivos e Capacidade Funcional em Idosos Institucionalizados. Cultura de los Cuidados (Edición digital), 19, 41.

VELOSO, M. V. et al. Functional Dependence in Institutionalized Elderly People and Memory Deficit. Revista ibero-americana de saúde e envelhecimento. vol. 2 n. ${ }^{\circ} 3$ dezembros 2016.

\section{Como citar este artigo (Formato ABNT):}

SILVA, Camila S.; SAMPAIO, Lucas S.; Sampaio, Talita S.O. Capacidade funcional de idosos em instituição de longa permanência. Id on Line Revista Multidisciplinar e de Psicologia, 2017, vol.11, n.38, p. 910-919. ISSN: 1981-1179.

Recebido: 11.11.2017

Aceito: 13.11.2017 